\title{
The Pediatric Gastrointestinal Tract: What Every Radiologist Needs to Know
}

\author{
Emily A. Dunn, Øystein E. Olsen, \\ and Thierry A. G. M. Huisman
}

\section{Learning Objectives}

- To compare imaging modalities commonly used in the assessment of pediatric gastrointestinal disease.

- To understand gastrointestinal pathology affecting children of different age groups.

- To recognize classic imaging features of gastrointestinal diseases commonly encountered in infants and children.

\subsection{Imaging Techniques}

\subsubsection{Plain Film Radiography and Fluoroscopy}

Conventional radiography remains the first-line imaging in most hospitals for infants and children presenting with suspected gastrointestinal pathology.

Fluoroscopy is particularly useful when evaluating dynamic processes such as gastrointestinal motility. Fluoroscopy can also be used for guiding procedures/interventions such as foreign body removal and intussusception reduction.

E. A. Dunn $(\bowtie)$ · Thierry A. G. M. Huisman

Division of Pediatric Radiology, Russell H. Morgan Department of Radiology and Radiological Science, Johns Hopkins University School of Medicine, Charlotte R. Bloomberg Children's Center, Baltimore, MD, USA

e-mail: Edunn18@jhmi.edu; Thuisma1@jhmi.edu

$\emptyset$. E. Olsen

Radiology Department, Great Ormond Street Hospital for Children NHS Foundation Trust, London, UK

e-mail: oystein.olsen@gosh.nhs.uk

\subsubsection{Ultrasound}

Ultrasound (US) is one of the most valuable imaging tools for the initial evaluation of gastrointestinal disease, due to its high-resolution, real-time capability, wide availability and acceptance, and lack of ionizing radiation and because it rarely requires sedation. There are two key factors for success: (1) knowing how to deal with children of all ages and (2) using high-end US equipment/high-frequency probes.

\subsubsection{Computed Tomography}

In children, CT should be used with caution and is usually reserved for troubleshooting equivocal findings made in the initial diagnostic workup of acutely and critically sick children. CT is consequently often performed as first-line imaging for emergent indications, such as blunt and penetrating trauma. As a general rule, only one acquisition is needed, and multiphase contrast enhanced sequences should be avoided.

\subsubsection{Magnetic Resonance Imaging}

MRI is rarely used in the first-line evaluation of gastrointestinal pathology; however, it may serve as an adjunct in challenging/unequivocal cases. In children, MRI is typically performed in the assessment of intestinal pathology such as inflammatory bowel disease and appendicitis, focal and diffuse disease of the abdominal viscera, and diseases of the biliary tree.

\subsection{Intestinal Obstruction}

In neonates and young children, there is no radiographic "signature" for the large and small bowel. Plain film radiographs of the normal newborn demonstrate swallowed air 
reaching the rectum by $24 \mathrm{~h}$ of life. In a clinically obstructed newborn, the number of dilated loops ("dilated" meaning roughly wider than the vertebral interpedicular space) may be more helpful. Whether there is one gas bubble (gastric outlet obstruction), a double bubble (duodenal obstruction), 3-4 bubbles/loops (other high small bowel obstruction), or more (low obstruction), the bowel gas pattern can help decide which contrast study is appropriate. A bowel gas pattern suspicious for a high obstruction may be assessed further with an upper GI contrast study. A bowel gas pattern suggesting a low obstruction should be followed by a contrast enema.

\subsection{Neonatal Obstruction}

Neonatal intestinal obstruction can be classified by location, with "high" obstructions occurring through the level of the proximal jejunum and "low" obstructions occurring after the proximal jejunum. Etiologies of neonatal intestinal obstruction are commonly congenital. In most cases, a fluoroscopic contrast study is required.
The differential diagnosis for high intestinal obstruction includes atresia of the esophagus, stomach, duodenum and jejunum, duodenal stenosis from annular pancreas, duodenal web, and malrotation possibly complicated by midgut volvulus.

The differential diagnoses for low intestinal obstruction include ileal and colonic atresia, anorectal malformation, meconium ileus, colonic dysmotility syndromes, and Hirschsprung's disease. Congenital abdominopelvic cysts or masses can cause both high and low (usually partial) obstruction.

An understanding of clinical presentation as well as the expected postnatal bowel gas progression throughout the abdomen on radiographs can help to detect the level of obstruction [1].

Esophageal atresia (Fig. 15.1) is a clinical diagnosis. If the diagnosis is established, then any gas in the gastrointestinal system distal to the esophagus on plain radiographs means there is also a tracheoesophageal fistula. Radiology has a greater role in the follow-up of these children. Fistulae may recur, and the contrast esophagogram is the imaging test of choice for diagnosis of recurrence; this is performed by trained operators as there is a considerable aspiration risk.
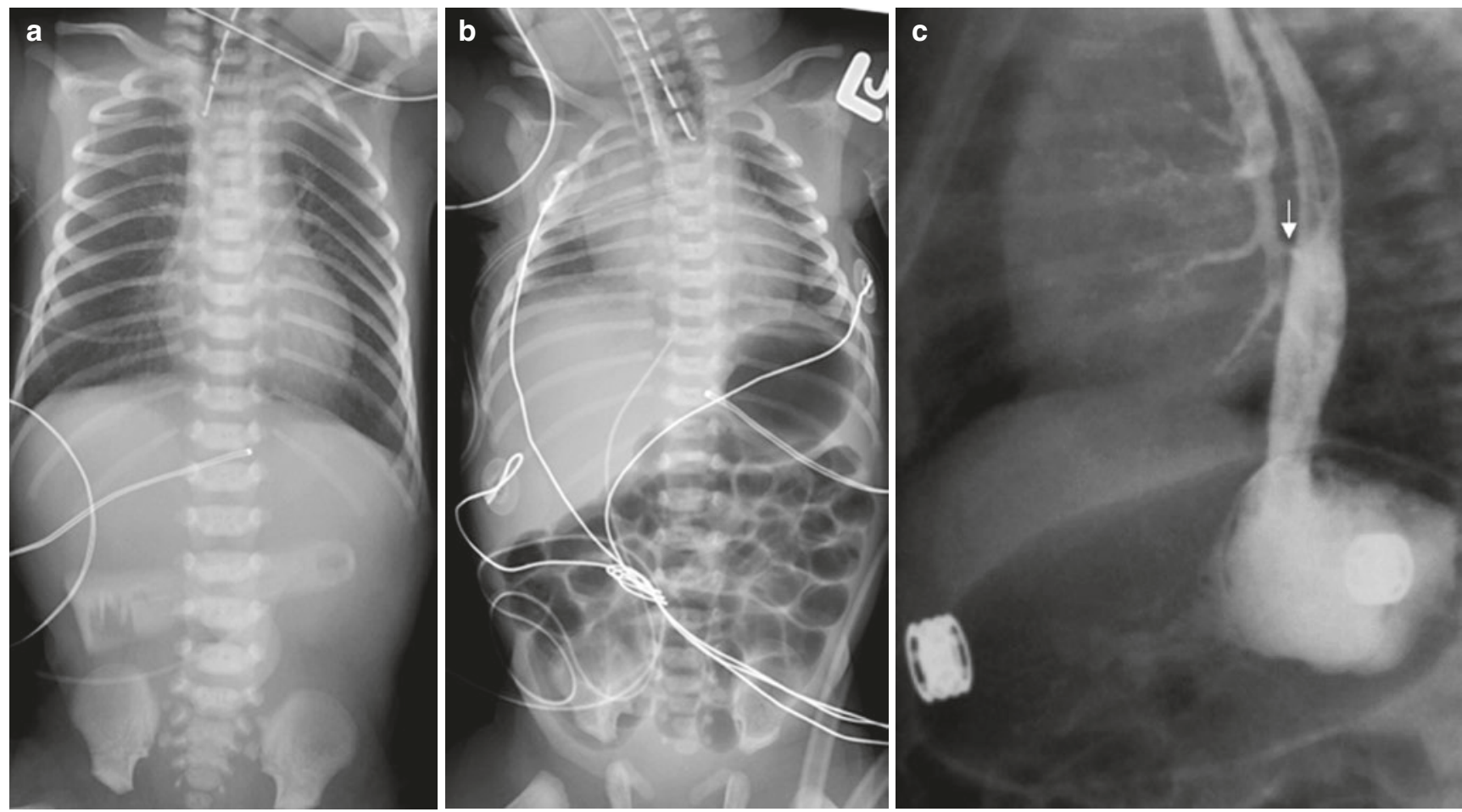

Fig. 15.1 Esophageal atresia and tracheoesophageal fistula represent a spectrum of anomalies believed to result from abnormal formation and separation of the embryological foregut. These anomalies range from isolated EA to isolated TEF. Classification of congenital tracheoesophageal anomalies depends on the presence and location of the fistulous communication between both structures. (a) Radiograph of a newborn with isolated esophageal atresia (no fistula) shows no bowel gas distally. Those with isolated EA may have polyhydramnios in utero, and at birth, there is failure of passage of the orogastric tube beyond the level of the atresia. (b) Radiograph of a newborn with esophageal atresia and a fistula between the airway and the distal atretic segment demonstrates gas in the gastrointestinal tract despite failure to pass a feeding tube. (c) Fluoroscopic image in an infant with isolated tracheoesophageal fistula (arrow) following instillation of water-soluble contrast medium in the esophagus 

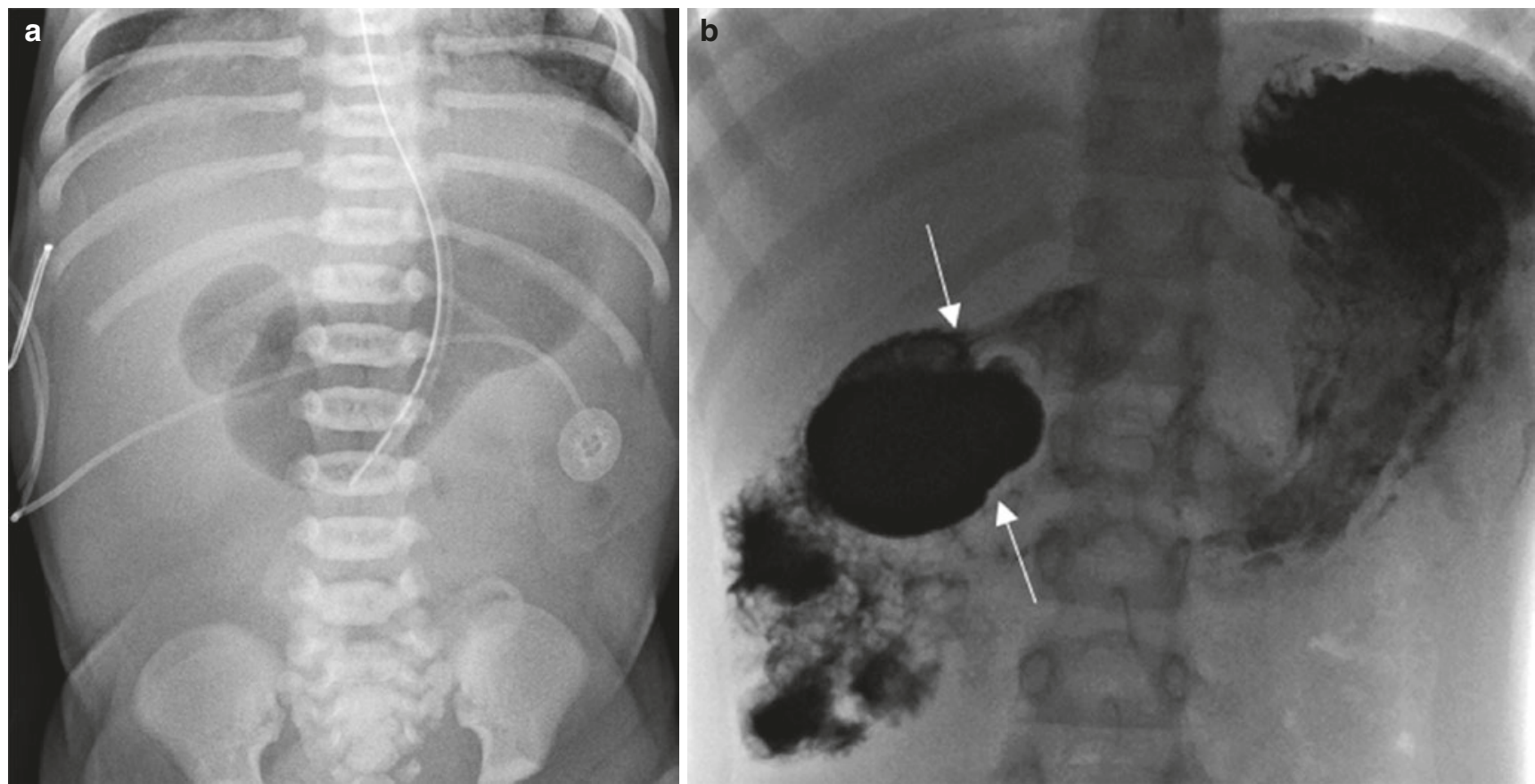

Fig. 15.2 Duodenal obstruction (complete or partial) is commonly caused by duodenal atresia, duodenal stenosis, duodenal web, annular pancreas, and midgut volvulus. (a) Radiograph of a 12-hour-old newborn with prenatally diagnosed duodenal atresia. Abdominal radiograph shows the "double bubble" appearance of the air-distended stomach and proximal duodenum and no gas distally. These findings suggest duodenal obstruction. It should be noted that annular pancreas and midgut volvulus may produce a similar radiographic appearance. (b) Newborn with vomiting. The first and second portions of the duodenum are distended. A curvilinear filling defect is observed at the transition point (arrows), representing the "windsock" appearance of the duodenal web
For suspected anastomotic stricture, contrast swallow is performed. There is almost always relative narrowing at the anastomosis, so it is a functional holdup which suggests a potential need for dilatation [2,3]. Esophageal atresia may be observed as part of the VACTERL association. Additional anomalies should be actively searched for.

Duodenal obstruction (Fig. 15.2) may have several causes. In duodenal atresia the classic radiographic findings are a double bubble and no distal intestinal gas. Duodenal atresia is thought to result from failure or incomplete recanalization of the intestine in utero.

Annular pancreas is caused by failed migration of the anlagen, and it may be evident sonographically (or with CT/MRI). Duodenal stenosis and duodenal web cause partial obstruction and may be difficult to distinguish. The classic sign of a web on a contrast study is the "windsock sign" $[4,5]$.

Malrotation with midgut volvulus (Fig. 15.3) is a surgical emergency in the neonate as well as in infants and children. The failed intestinal rotation manifests as a right-sided/midline low duodenojejunal flexure and a high cecum. This is associated with a short mesenteric root, which predisposes for volvulus. The normal position of the duodenojejunal flexure is at the height of the pylorus and to the left of the midline, at least as far as the left vertebral pedicles. Volvulus is suggested by bilious vomiting and is verified by the finding of a spiraling outline of a malrotated duodenum/proximal jejunum on the contrast study [6,7]. On US a whirlpool of vessels is typically seen.

Small bowel atresia and stenosis (Fig. 15.4) are relatively common causes of complete and incomplete obstruction in the neonate. Multiple atresias may coexist; the etiology is thought to be in utero mesenteric vascular accidents. Accurate diagnosis is critical, since early surgical invention is usually performed. Bilious vomiting may be observed in those with obstruction beyond the level of the pancreatic ampulla. The typical findings of atresia on contrast enema are a small-caliber (unused) colon and distal small bowel and the inability to reflux contrast into dilated small bowel loops (distinguishing it from meconium ileus; see below) [4, 5].

Meconium ileus (Fig. 15.5) has a very high association $(>80 \%)$ with cystic fibrosis. The distal ileum is impacted with sticky meconium. The findings on contrast enema are similar to those in small bowel atresia except that when contrast refluxes into the ileum, it outlines pellets of meconium, and it is usually possible to eventually fill dilated small bowel loops. In uncomplicated cases, (repeated) contrast enemas using water soluble contrast material are used therapeutically to soften and wash out meconium pellets. Both meconium ileus and intestinal atresias may be complicated by in utero bowel necrosis/perforation and meconium peritonitis $[8,9]$. 


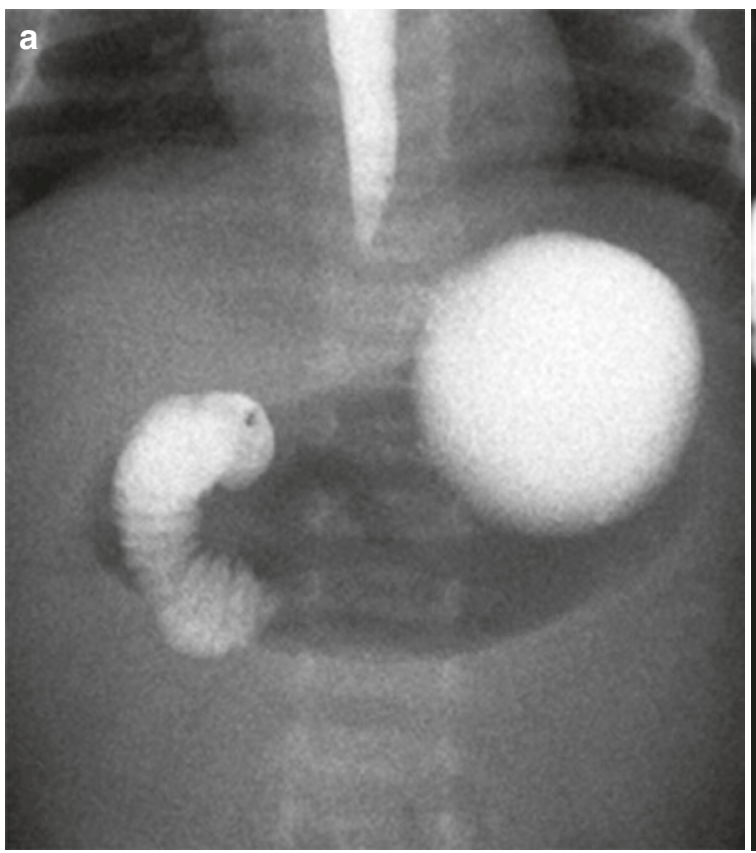

Fig. 15.3 Intestinal malrotation results from incomplete rotation and fixation of the bowel in utero, with the duodenojejunal junction and cecum lying closer. This shortening of the mesenteric pedicle predisposes to midgut volvulus, in which the abnormally fixed bowel loops twist about the axis of the superior mesenteric artery and may obstruct the duodenum. While malrotation with midgut volvulus may occur at any age, the great majority present within their first month and many within their first week. Midgut volvulus can lead to intestinal ischemia necessitating bowel resection. Emergent and accurate imaging diagnosis is therefore critical. Bilious vomiting and abdominal distention in a

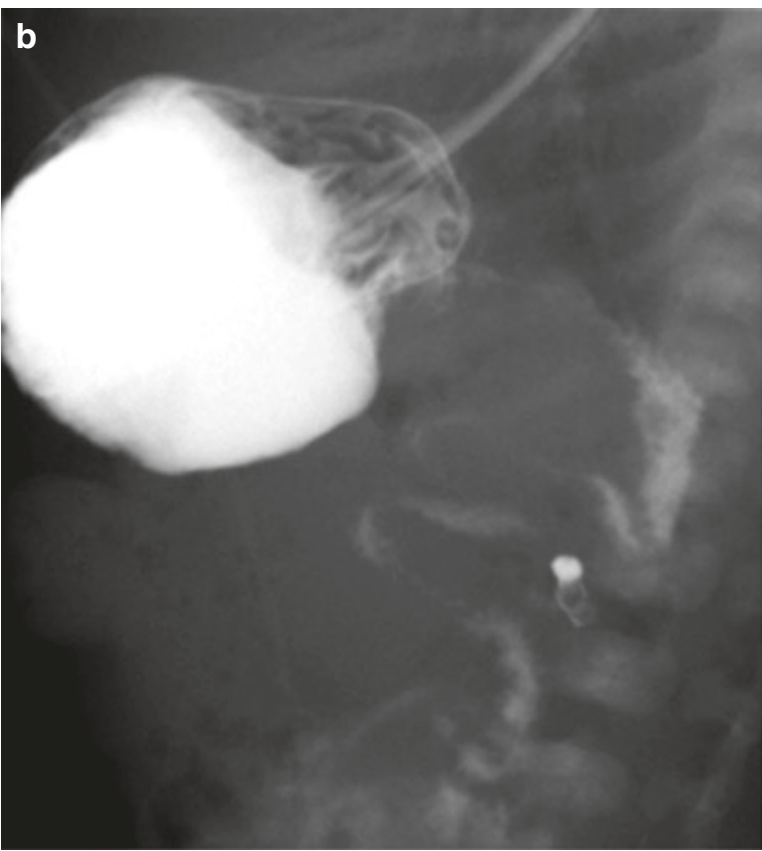

newborn infant should raise the suspicion for an obstruction distal to the ampulla of Vater, and imaging should be pursued. Radiographs are normal in many cases of malrotation, and a fluoroscopic upper gastrointestinal series, which is considered the gold standard for diagnosis, should be performed in highly suspect cases. (a) Malrotation with midgut volvulus. In the case of malrotation with midgut volvulus, contrast material fails to pass beyond the obstructed third portion of the duodenum. (b) Malrotation with midgut volvulus in the setting of an incomplete obstruction. There is corkscrew configuration of contrast within the twisted midgut bowel loops
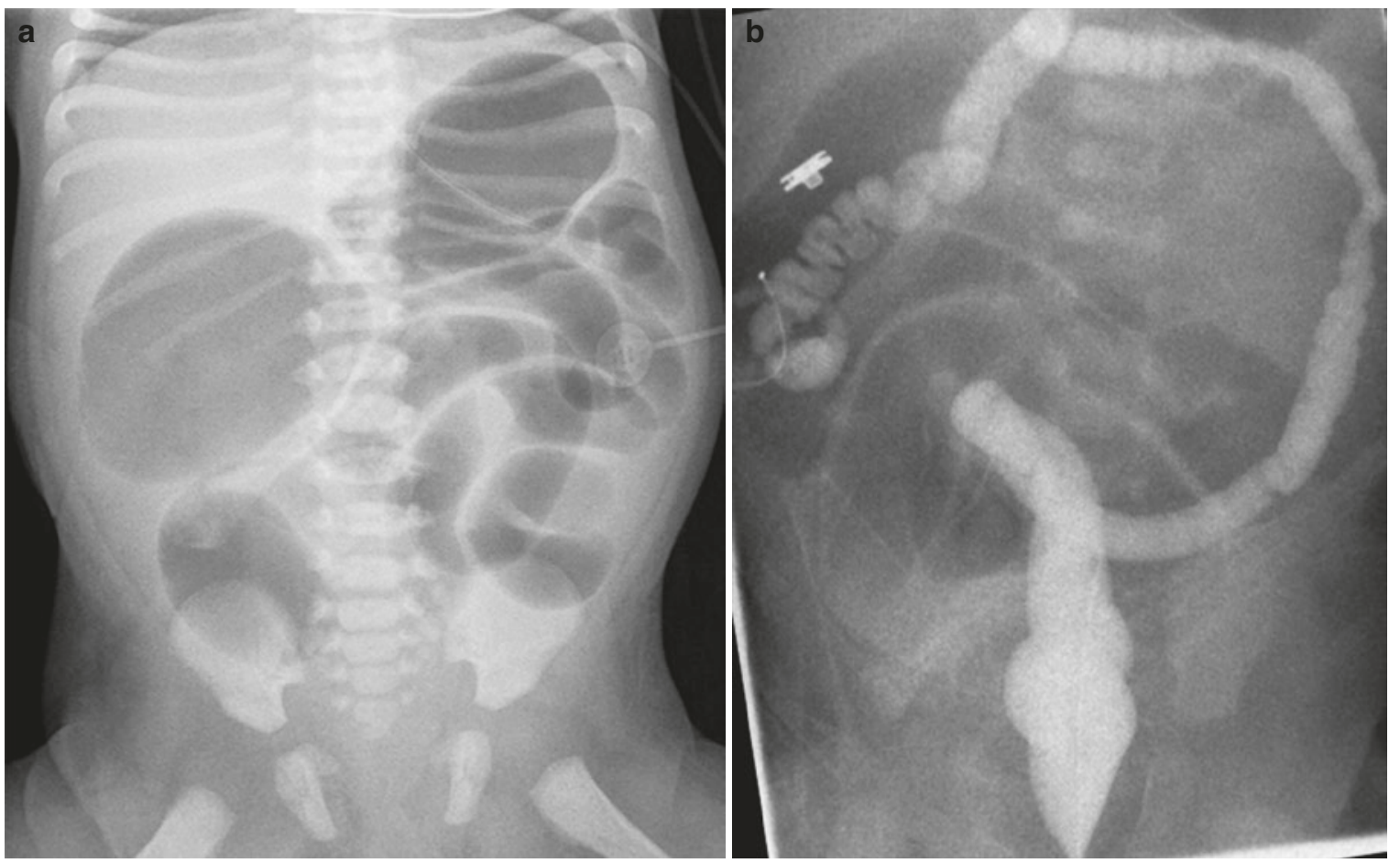

Fig. 15.4 Ileal atresia is thought to result from mesenteric vascular events in utero. (a) Radiograph of a newborn infant with ileal atresia shows distention of multiple bowel loops throughout the abdomen, suggesting a low obstruction. (b) Fluoroscopic contrast enema in the same child demonstrates a typical "microcolon" (unused colon) and retrograde filling of non-dilated distal ileum to the level of the atresia (arrow). More proximal bowel loops are distended and air filled 


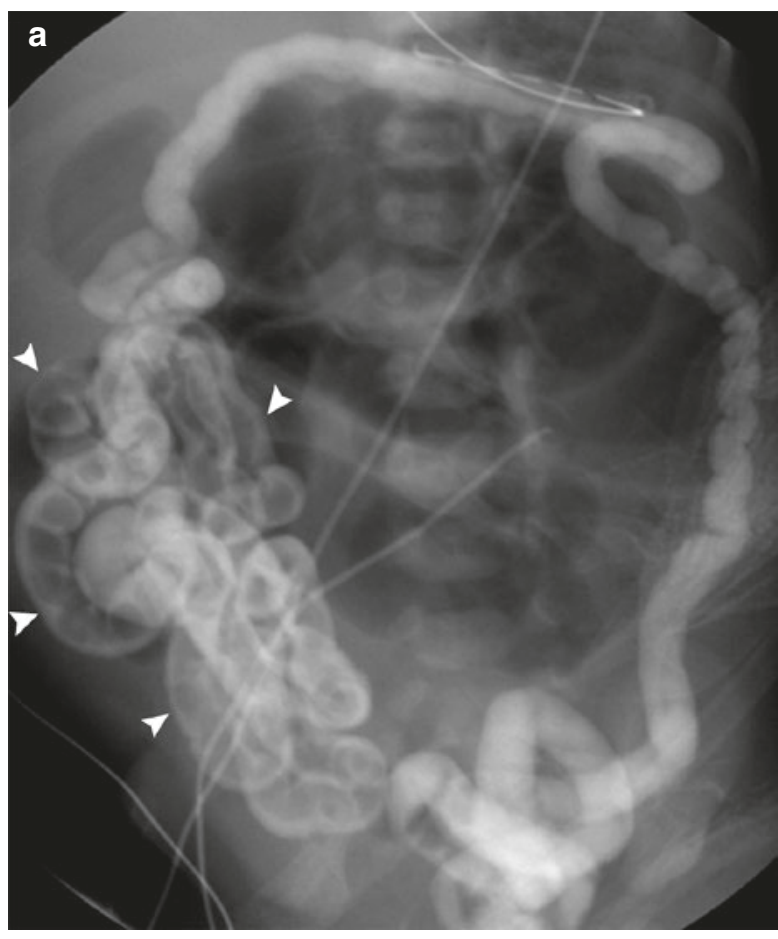

Fig. 15.5 Meconium ileus represents obstruction of the distal ileum by viscous, impacted meconium pellets. There is a high association with cystic fibrosis (presenting symptom in $15 \%$ of neonates with CF). (a) Fluoroscopic contrast enema demonstrates contrast opacification of a "microcolon" (unused colon due to proximal obstruction). Contrast has refluxed into the

Colonic atresia is exceedingly rare, and the diagnosis is often made clinically (vomiting and failure to pass meconium) on plain radiographs (no gas in the rectum). Contrast enema reveals failed reflux of contrast proximal to the level of the colonic atresia [10].

In Hirschsprung's disease (Fig. 15.6), intermuscular and submucosal nerve plexuses are absent due to arrested migration of intestinal ganglion cells, producing a functional intestinal obstruction. Since this migration occurs from proximal to distal large bowel, the aganglionic segment extends proximally from the anus. The typical clinical presentation is delayed passage of meconium in the neonate beyond $24 \mathrm{~h}$ of age.

Most affected patients demonstrate short-segment involvement, in which the transition point between normal and aganglionic bowel is located in the rectosigmoid colon. Rarely, the entire colon may be involved. Meconium/fecal impaction, a zone of transition from dilated to non-dilated bowel, and abnormal peristalsis of the affected colon segment with "sawtooth" appearance of the wall are classical findings at contrast enema. Very short-segment aganglionosis lacks a clear transition zone and is therefore difficult to diagnose radiologically. Hirschsprung's disease can only be definitively diagnosed by rectal (suction) biopsy [4, 11].

Functional immaturity of the colon, small left colon, and meconium plug syndrome (Fig. 15.7) are entities with considerable overlap. The risk is greater in babies of diabetic

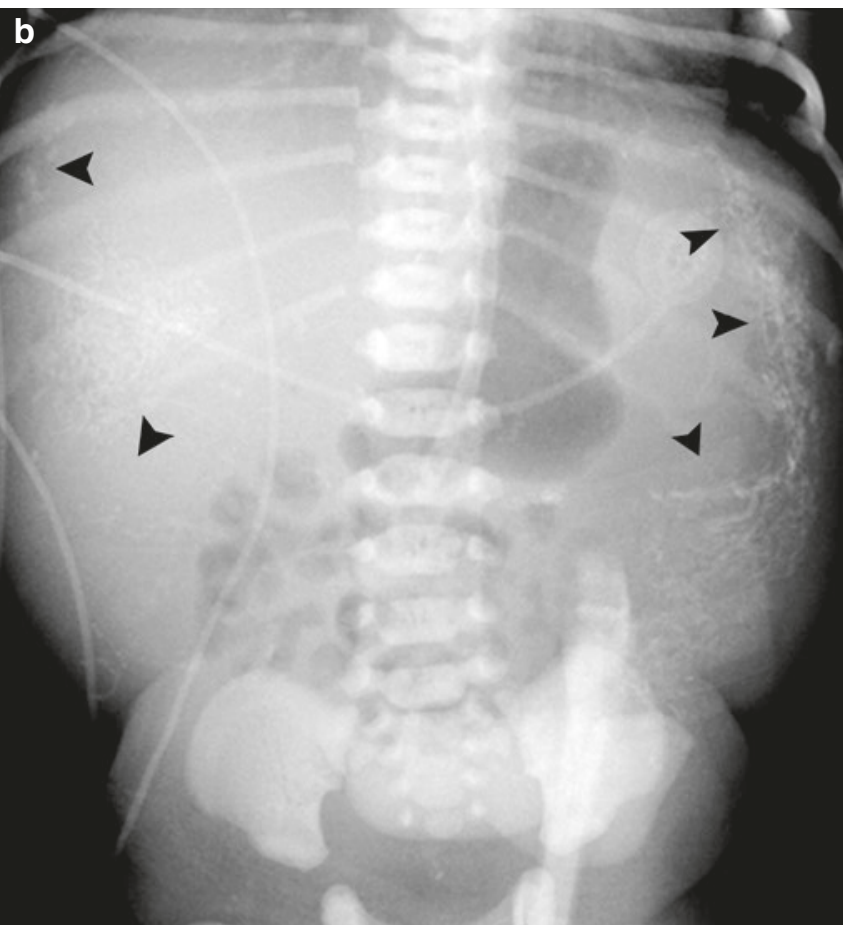

distal ileum (arrowheads) and outlines numerous impacted meconium pellets. (b) Abdominal radiograph demonstrates punctate calcifications scattered throughout the abdomen, suggestive of meconium peritonitis which can occur with intestinal atresias as well as meconium ileus, and results from perforation of meconium-containing bowel

mothers. Radiologically there is a relatively smaller left colon and proximal meconium impaction. It is difficult to make a categorical diagnosis as similar findings may be seen in Hirschsprung's disease [12, 13].

Anorectal malformation is a clinical diagnosis. These malformations are classified as "high" or "low" depending on the location of the distal-most bowel segment relative to the puborectalis sling, with high lesions terminating above and low lesions terminating below this level. The role of radiology is to assess the length of the atretic anorectal segment and to map the fistula(e) [14].

\subsection{Intestinal Obstruction in the Older Neonate and Infant}

In older infants and children, obstruction may be related to other etiologies, such as adhesions, hypertrophic pyloric stenosis, intussusception, incarcerated intestinal hernia, appendicitis, sigmoid volvulus, and Meckel's diverticulum. Plain film radiographs can occasionally suggest the level of obstruction in older infants and children. In many cases, however, ultrasound and, occasionally, CT or MRI may be necessary to reach the diagnosis.

Hypertrophic pyloric stenosis (Fig. 15.8) classically presents with forceful "projectile" vomiting and failure to gain weight in an infant. The diagnosis is made sonographically 


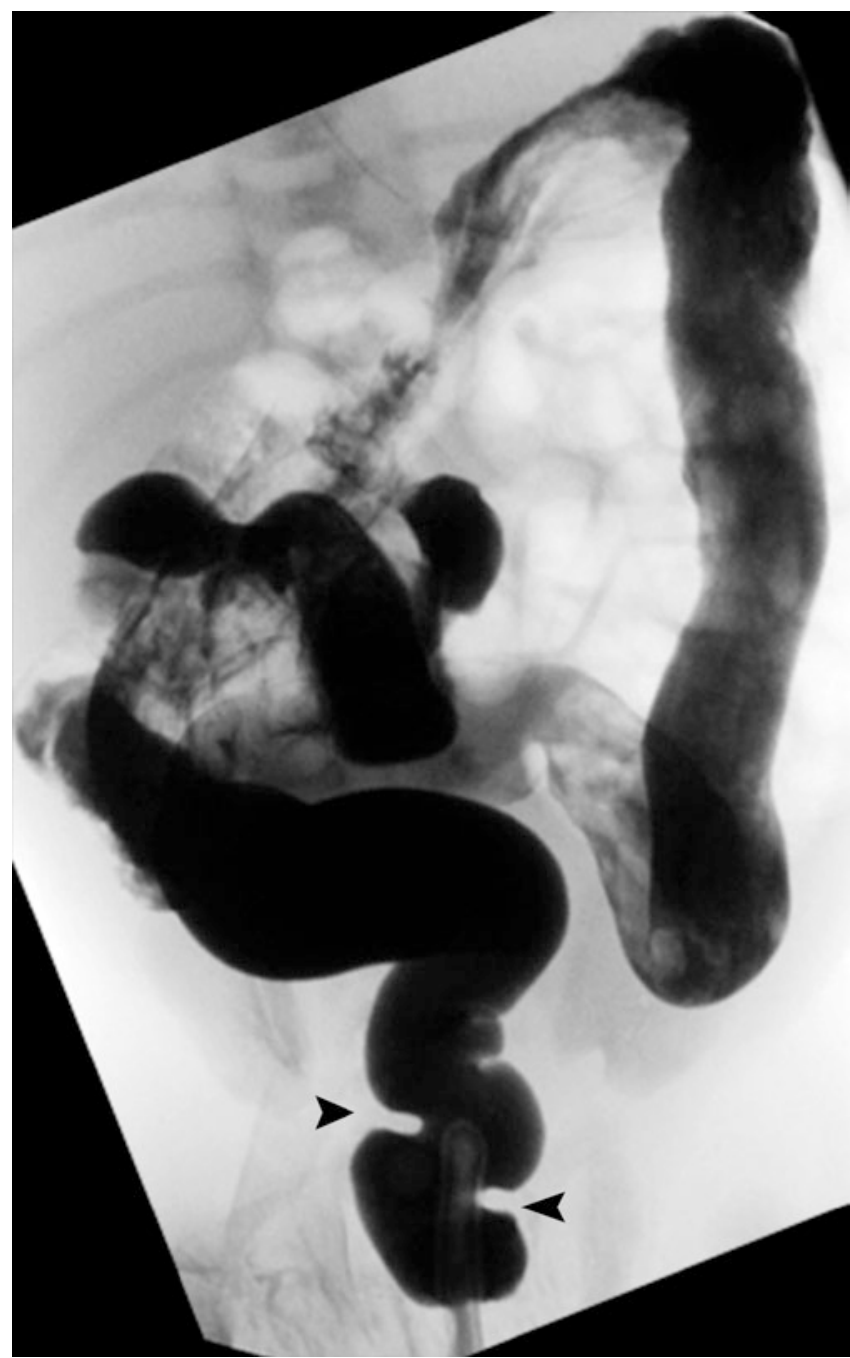

Fig. 15.6 A fluoroscopic contrast enema in a 2-day-old with biopsyproven Hirschsprung's disease shows a small-caliber rectum with mild distention of more proximal large bowel loops. Note the "sawtooth" appearance of the aganglionic segment (arrowheads), which reflects irregular contractions

where the typical findings are (1) thickened muscularis (>4 mm); (2) elongated pylorus ( $>15 \mathrm{~mm})$, with redundant mucosa occasionally protruding into the antrum of the stomach; (3) gastric hyperperistalsis; and (4) persistent closed pyloric channel after a test feed. A contrast meal is rarely indicated except in a few equivocal cases [15].

Incarcerated intestinal hernia is a surgical emergency. The presentation and imaging findings are similar to those in adults.

Congenital abdominopelvic masses (Fig. 15.9) comprise a spectrum of abnormalities where gastrointestinal obstruction is relatively unusual. Sacrococcygeal masses (e.g., germ cell tumors, such as teratomas, or anterior meningoceles) may cause rectal obstruction. A cystic obstructive mass should raise suspicion for meconium pseudocyst or enteric duplication cyst. Duplication cysts may occur anywhere along the gastrointestinal tract and may have a relatively thick layered wall (akin to bowel) and a mucus-fluid level.

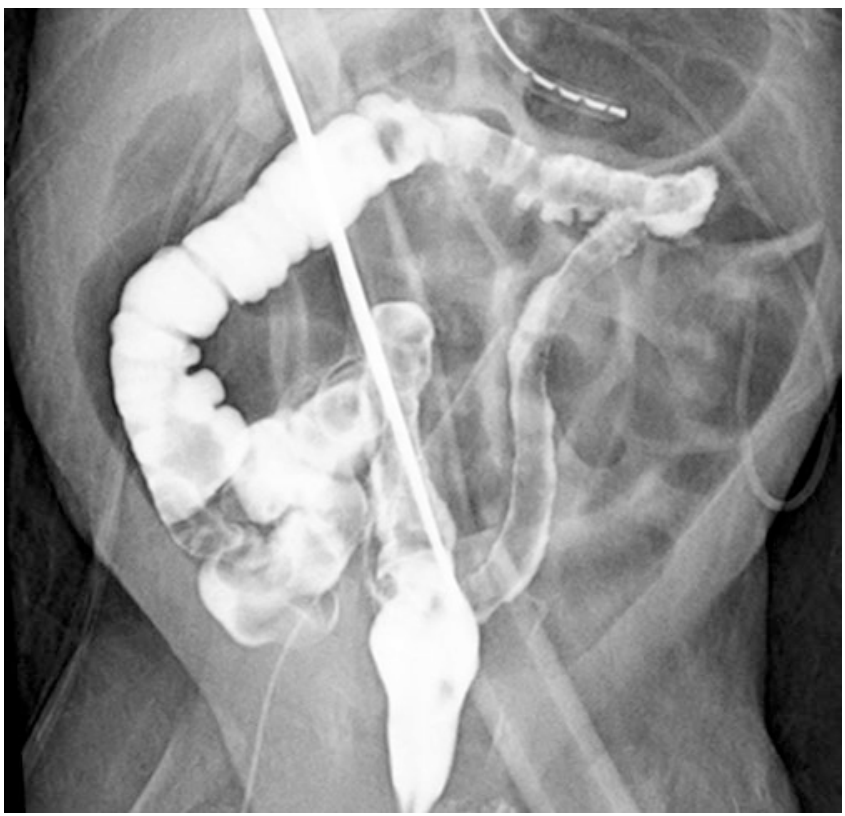

Fig. 15.7 Functional immaturity of the colon, along with meconium plug and small left colon, represent syndromes of colonic dysmotility. Those affected typically present with delayed passage of meconium. There is a reported increased incidence in babies with mothers who are diabetic or who have received magnesium sulfate during pregnancy for preeclampsia. This image from a contrast enema reveals a small caliber of the descending and sigmoid colon, which are filled with meconium, proximal dilatation, and a normal-caliber rectum

Differential diagnoses for abdominal cystic lesions that are rarely obstructive include choledochal, mesenteric, and ovarian cysts, renal cystic dysplasia, and hydroureter [16].

\subsection{Intestinal Obstruction in the Infant and Older Child}

Ileocolic intussusception (Fig. 15.10) is a surgical emergency, usually occurring in infants and toddlers with maximum incidence at just under 1 year of age. The typical presentation is with abdominal pain, bloody stools, and a palpable mass. A clear cause (i.e., a "lead point," such as a mesenteric lymph node, lymphoid hyperplasia, duplication cyst, mural inflammation caused by Henoch-Schonlein purpura, or Meckel's diverticulum) may or may not be established. The sonographic diagnosis is fairly straightforward with the finding of a concentric doughnut shape in the transverse plane and sandwiching of the involved bowel segments (the "pseudokidney" sign) in the longitudinal plane [17].

Long-standing intussusception, trapped fluid in the involved segments, and trapped lymph nodes are thought to be associated with lower success rates for reduction with pressurized gas or fluid.

Colonic volvulus results from twisting of the colon around its mesenteric root and is a rare cause of obstruction in children. In children, cecal volvulus is more common compared with volvuli occurring in the sigmoid and transverse colon. 


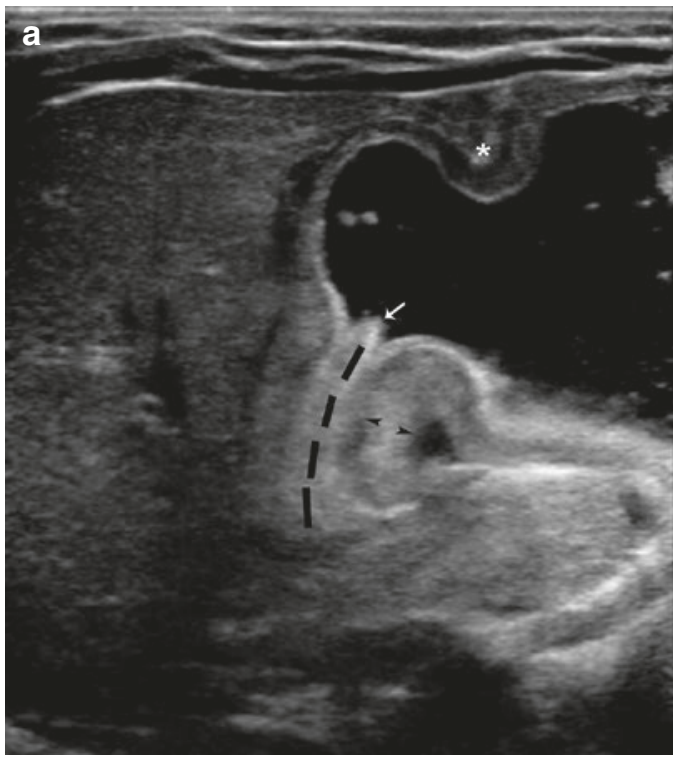

Fig. 15.8 Hypertrophic pyloric stenosis is an acquired obstruction of the gastric outlet, characterized by elongation of the antropyloric channel and thickening of the pyloric muscularis with varying degrees of mucosal hypertrophy. Infants typically present with non-bilious, projectile vomiting and occasionally a palpable hypertrophied pyloric "olive." Hypertrophic pyloric stenosis is usually not present at birth. Infants usually present with symptoms at $2-12$ weeks of age. The diagnosis is made sonographically. (a) High-frequency ultrasound examination of the antropyloric region shows thickening of the muscularis layer

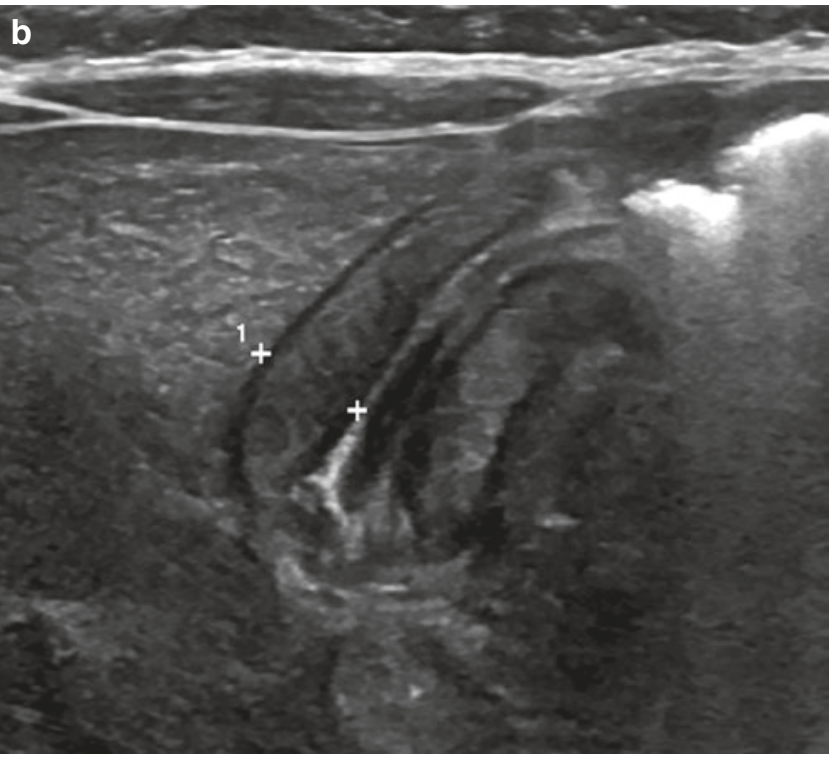

to more than $3 \mathrm{~mm}$ (arrowheads) and elongation of the pyloric channel to more than $15 \mathrm{~mm}$ in length (dashed line). Dynamic imaging can detect the presence or absence of transit through the pyloric channel and may also identify hyperperistaltic gastric waves (asterisk) in the presence of a gastric outlet obstruction. Redundant pyloric mucosa may protrude into the gastric lumen (arrow), producing the "antral nipple sign." (b) High-frequency detailed ultrasound often demonstrates striation within the thickened muscularis (between calipers)

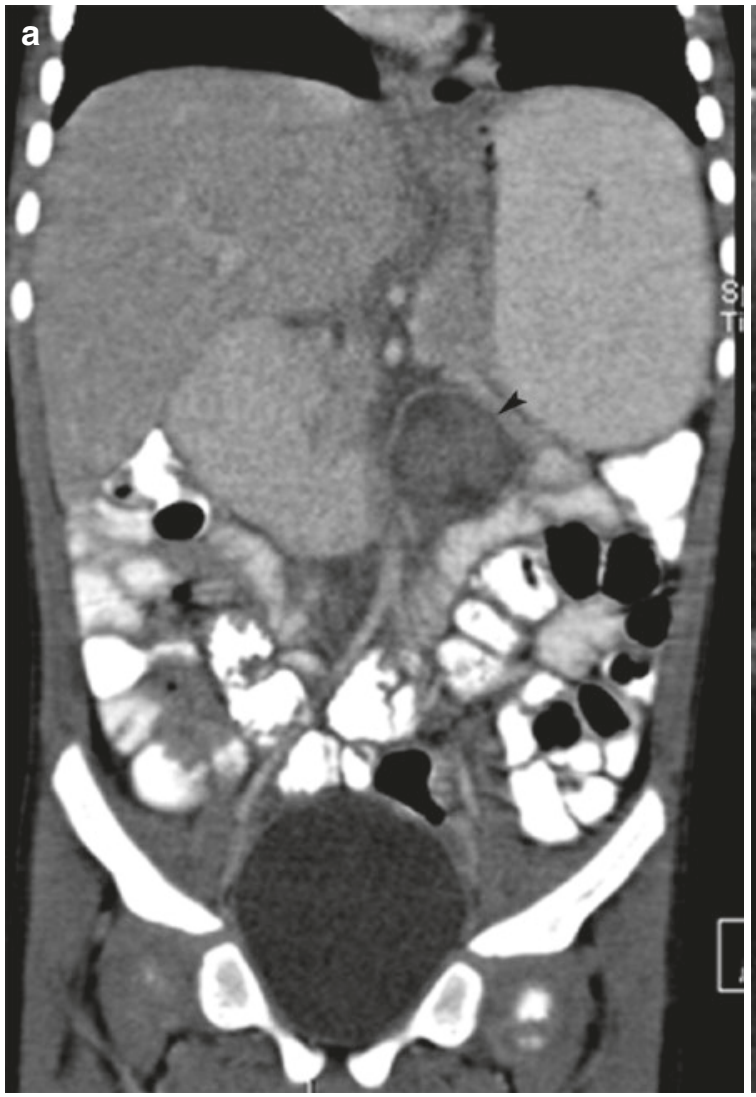

Fig. 15.9 Duplication cysts represent closed congenital duplicated segments of any part of the gastrointestinal tract and may be diagnosed incidentally or as a result of obstruction. (a) Coronal reformat of computed tomography scan following oral and intravenous contrast medium

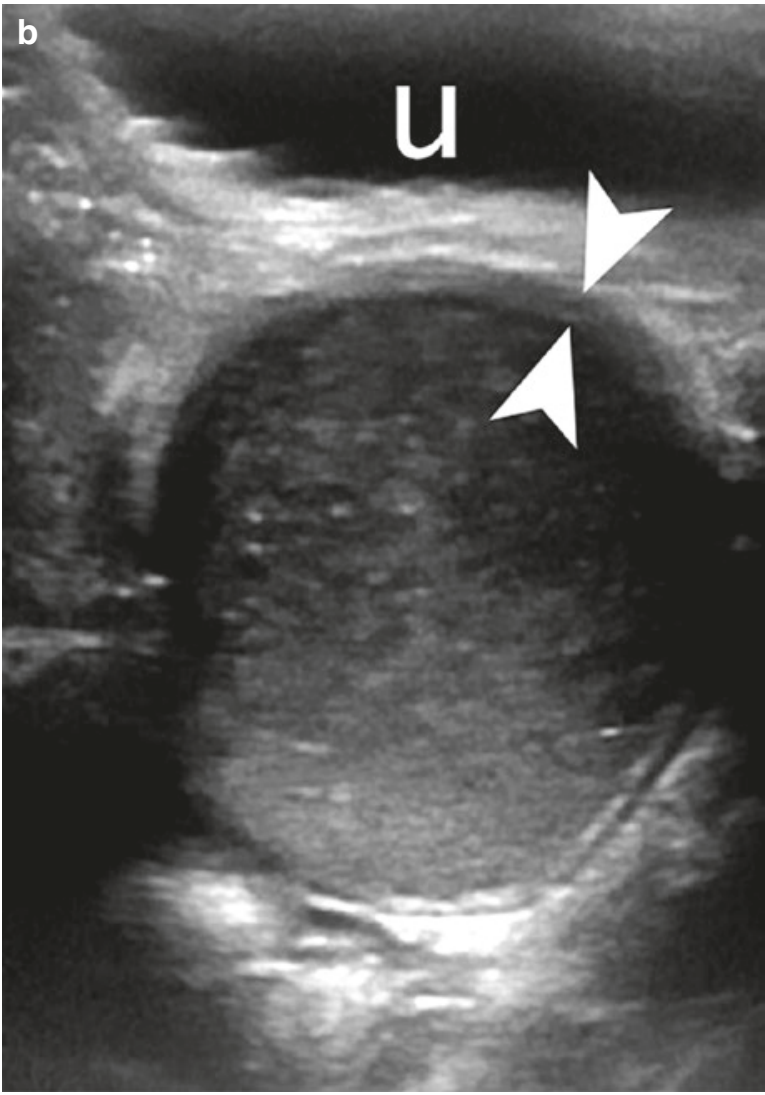

administration demonstrates a small bowel duplication cyst (arrowhead). (b) Sagittal sonogram shows a cyst posterior to the urinary bladder $(u)$. The diagnosis of a rectal duplication cyst is readily made by demonstrating a bowel wall appearance of the cyst wall (arrowheads) 

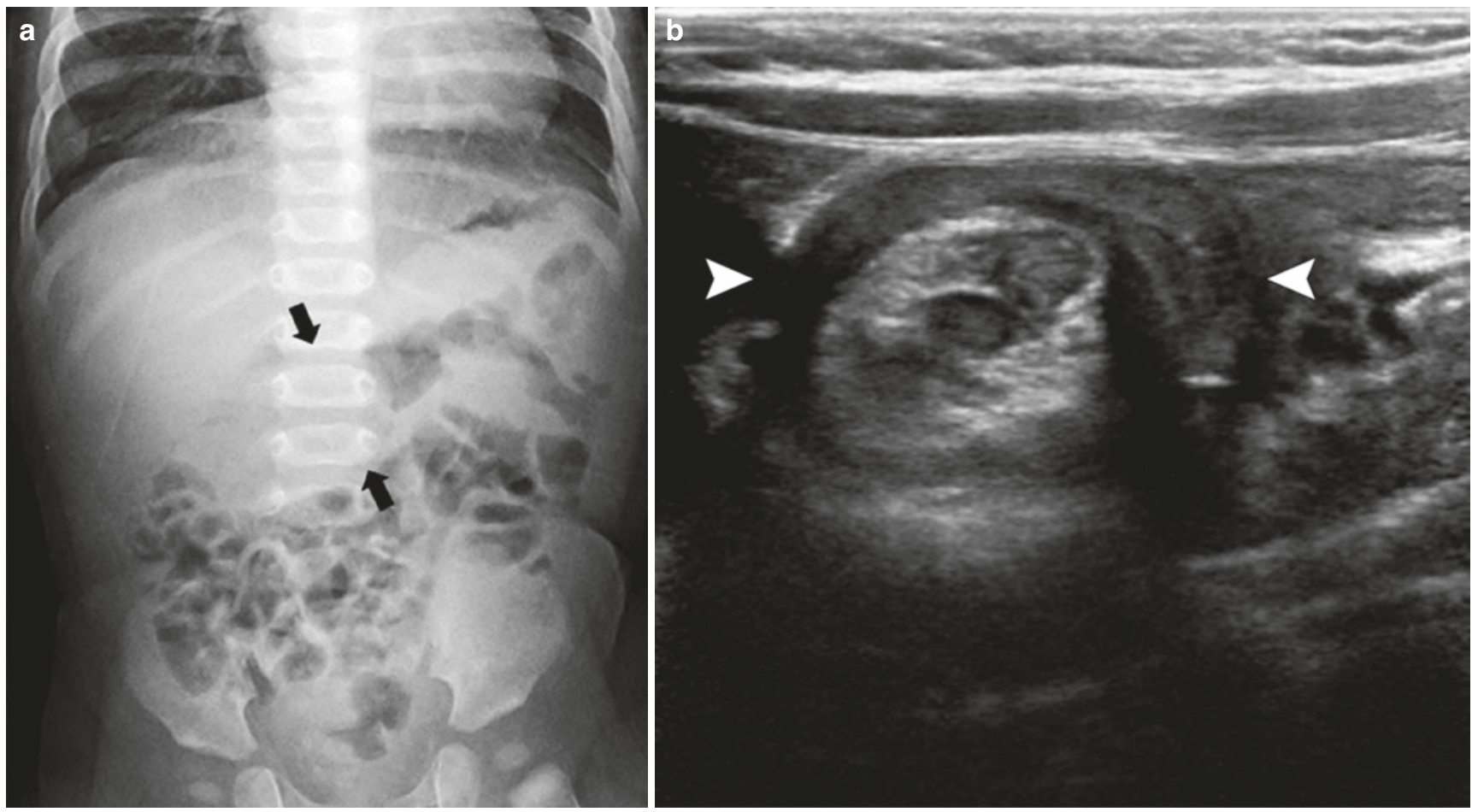

Fig. 15.10 Intussusception, most commonly ileocolic, has peak incidence in late infancy and presents with abdominal distention and bloody stools. (a) Radiographs may show, as in this example, a well-defined opacity (arrows) representing the invaginate. (b) Ultrasound is diagnos- tic showing a typical doughnut configuration (arrowheads) of the telescoping bowel segments with trapped mesenteric fat (hyperechoic content)
Clinical presentation includes abdominal pain, vomiting, and distention. Depending on the type of twist, plain films may demonstrate marked distention of the colon and/or air-fluid levels resembling either a "bird's beak" or "coffee bean." CT demonstrates the "swirling" appearance of the sigmoid mesentery and its vasculature. While clinical and imaging findings are suggestive, sigmoid volvulus remains an often missed diagnosis which can be complicated by bowel ischemia, gangrene, perforation, shock, and even death [18-20].

Henoch-Schonlein purpura is an acute small-vessel vasculitis in young children typically involving the skin, kidneys, synovium, and bowel. Sonographically there is nonspecific mural inflammation [21].

\subsection{Infectious, Ischemic, and Inflammatory Intestinal Pathology}

Necrotizing enterocolitis (Fig. 15.11) is the most common gastrointestinal emergency in the newborn. The etiology of NEC is incompletely understood and is thought to involve multiple factors, including immaturity of the blood-gut barrier, early feeding, and bacterial colonization which ultimately lead to hemorrhagic and ischemic necrosis of the intestines. NEC typically occurs in preterm neonates especially with very low birthweight and less commonly term or late preterm neonates with congenital cardiac disease or Hirschsprung's disease. Although somewhat insensitive, plain films may demonstrate intestinal pneumatosis, portal venous air, and pneumoperitoneum. Intestinal ultrasound has proven useful in the diagnosis of NEC and may demonstrate the presence of free fluid and abnormal bowel peristalsis, increased wall thickness, echogenicity, and vascularity and may suggest impending complications such as perforation. Additionally, US has been shown to detect small amounts of intramural and portal venous "air bubbles" not demonstrated on plain film [22, 23].

Acute appendicitis is the most common surgical indication in children. Appendicitis results from obstruction of the appendix which leads to distention, mucosal ischemia, bacterial overgrowth, inflammation, and potentially wall compromise and perforation. Early diagnosis is therefore essential in order to avoid such complications. US is the initial test of choice due to its lack of ionizing radiation and is optimal for imaging children. With an experienced sonographer and use of graded compression technique, US is highly sensitive and specific for the diagnosis of appendicitis and its complications and approaches the accuracy of CT. Use of MRI in the diagnosis of appendicitis is increasing, particularly for children with equivocal ultrasound [24, 25]. 


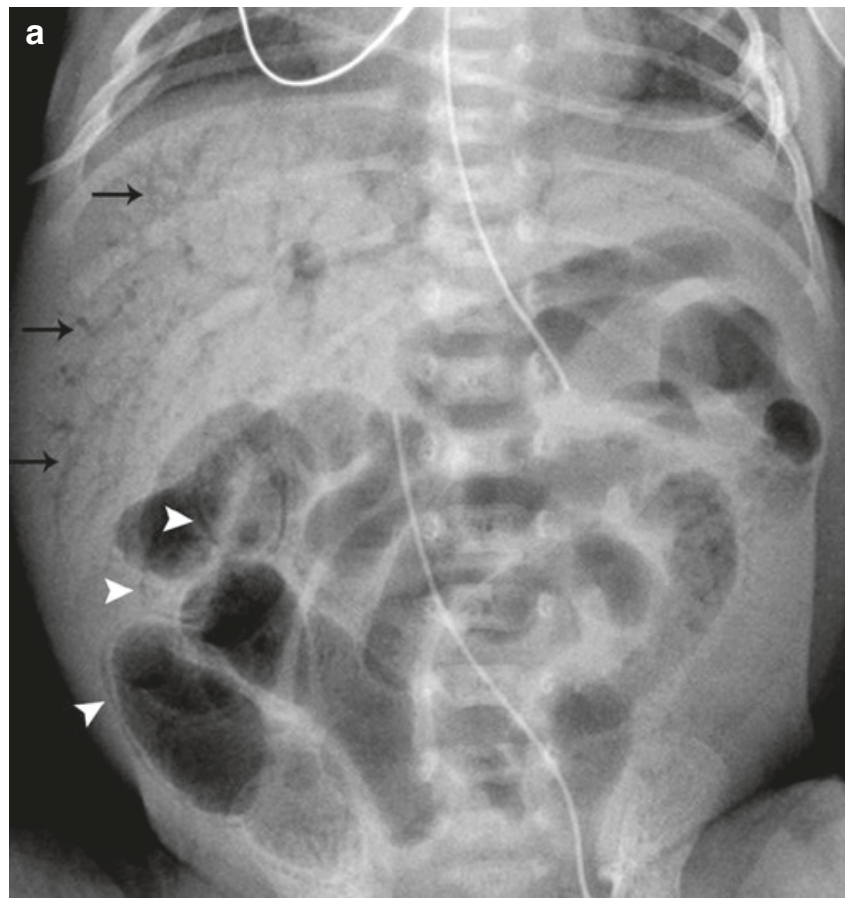

Fig. 15.11 Necrotizing enterocolitis is a clinical diagnosis but may nevertheless have typical radiological features. (a) Abdominal radiograph demonstrates thick-walled gas-distended bowel loops with intra-

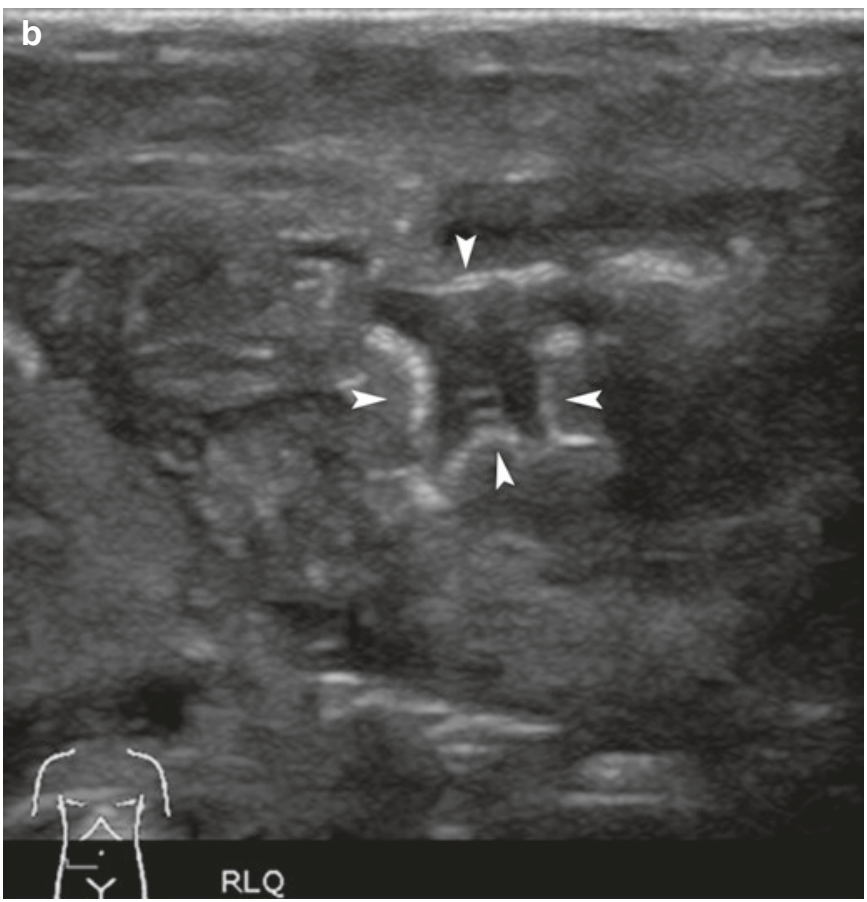

mural gas seen as linear lucencies (arrowheads) and portal venous gas (arrows). (b) Intramural gas demonstrated sonographically as a string of hyperechoic "pearls" (arrowheads) in the bowel wall
Inflammatory bowel disease may be seen at any age, but early-onset disease is often more complex with associated immunological disorders. In older children and adolescents, the clinical picture and the imaging characteristics are more similar to those in adults. Conventional fluoroscopic contrast follow-through is rarely indicated. Both ultrasound and MR enterography are excellent for assessing the macroscopic disease: bowel wall thickening, mesenteric inflammation, mesenteric lymphadenopathy, hyperemia, and fistulation [26].

Meckel's diverticulitis is inflammation of a Meckel's diverticulum, an omphalomesenteric remnant containing gastric/pancreatic mucosa. Abdominal pain and melena are typical symptoms. The diagnosis is extremely difficult to make with radiographs and cross-sectional imaging alone, which are often insensitive. $99 \mathrm{mTc}$-pertechnetate scintigraphy is typically diagnostic [27].

\subsection{Gastrointestinal Trauma}

Accidental and non-accidental traumas, particularly blunt trauma, are common causes of morbidity and mortality in children. Clinical and laboratory findings may suggest injury to the hollow and solid abdominal viscera, necessitating imaging follow-up. CT performed with intravenous contrast provides superb detail of vascular, solid organ, intestinal, and musculoskeletal anatomy, thus making it the imaging of choice in stable children presenting with trauma. US may be used in unstable patients and is especially helpful in the detection of hemoperitoneum. The liver, spleen, and kidneys are among the most common solid organ injuries observed in accidental trauma. In non-accidental trauma, the liver and pancreas are the most commonly injured solid organs. Intestinal injury is uncommon overall, yet occurs with more frequency in non-accidental versus accidental trauma.

Children with compensated shock following initial resuscitation efforts may demonstrate characteristic abdominal CT findings known as the hypoperfusion complex, which includes collapse of the aorta and inferior vena cava and hyperenhancement of the adrenals, kidneys, mesentery, and bowel walls. These findings signal impending hemodynamic collapse, and their immediate recognition is critical [28-30].

\section{Take-Home Messages}

- The differential diagnosis for neonatal high intestinal obstruction includes atresia of the esophagus, stomach, duodenum and jejunum, duodenal stenosis from annular pancreas, duodenal web, and malrotation with midgut volvulus. 
- The differential diagnoses for neonatal low intestinal obstruction include ileal and colonic atresia, anorectal malformation, meconium ileus, colonic dysmotility syndromes, and Hirschsprung's disease.

- Congenital abdominopelvic cysts may lead to high or low intestinal obstruction.

- In older infants and children, intestinal obstruction may be related to adhesions, pyloric stenosis, intussusception, incarcerated intestinal hernia, appendicitis, sigmoid volvulus, and Meckel's diverticulum.

- Radiographic findings of NEC including free, intramural, and portal venous air are classic yet infrequently observed. Ultrasound improves sensitivity.

- In the hands of an experienced sonographer and with use of graded compression technique, sensitivity and specificity of intestinal ultrasound for acute appendicitis approaches that of CT.

- Contrast enhanced CT is the imaging standard in the evaluation of stable children with blunt or penetrating trauma.

\section{References}

1. Grob M. Intestinal obstruction in the newborn infant. Arch Dis Child. 1960;35:40-50.

2. Harmon C, Coran A. Congenital anomalies of the esophagus. In: Coran A, editor. Pediatric surgery. Philadelphia: Elsevier; 2012. p. 893-918.

3. Cumming WA. Neonatal radiology. Esophageal atresia and tracheoesophageal fistula. Radiol Clin N Am. 1975;13(2):277-95.

4. Hernanz-Schulman M. Imaging of neonatal gastrointestinal obstruction. Radiol Clin N Am. 1999;37(6):1163-86, vi-vii.

5. Berrocal T, Torres I, Gutierrez J, Prieto C, del Hoyo ML, Lamas M. Congenital anomalies of the upper gastrointestinal tract. Radiographics. 1999;19(4):855-72.

6. Millar AJ, Rode H, Cywes S. Malrotation and volvulus in infancy and childhood. Semin Pediatr Surg. 2003;12(4):229-36.

7. Torres AM, Ziegler MM. Malrotation of the intestine. World J Surg. 1993;17(3):326-31.

8. DeLorimier AA, Fonkalsrud EW, Hays DM. Congenital atresia and stenosis of the jejunum and ileum. Surgery. 1969;65(5):819-27.

9. Allan JL, Robbie M, Phelan PD, Danks DM. Familial occurrence of meconium ileus. Eur J Pediatr. 1981;135(3):291-2.

10. Etensel B, Temir G, Karkiner A, Melek M, Edirne Y, Karaca I, et al. Atresia of the colon. J Pediatr Surg. 2005;40(8):1258-68.

11. StranzingerE, DiPietro MA, TeitelbaumDH, Strouse PJ. Imaging of total colonic Hirschsprung disease. Pediatr Radiol. 2008;38(11):1162-70.
12. Siddiqui MM, Drewett M, Burge DM. Meconium obstruction of prematurity. Arch Dis Child Fetal Neonatal Ed. 2012;97(2):F147-50.

13. Ellis H, Kumar R, Kostyrka B. Neonatal small left colon syndrome in the offspring of diabetic mothers-an analysis of 105 children. J Pediatr Surg. 2009;44(12):2343-6.

14. Berrocal T, Lamas M, Gutieerrez J, Torres I, Prieto C, del Hoyo ML. Congenital anomalies of the small intestine, colon, and rectum. Radiographics. 1999;19(5):1219-36.

15. MacMahon B. The continuing enigma of pyloric stenosis of infancy: a review. Epidemiology. 2006;17(2):195-201.

16. Khong PL, Cheung SC, Leong LL, Ooi CG. Ultrasonography of intra-abdominal cystic lesions in the newborn. Clin Radiol. 2003;58(6):449-54.

17. Applegate KE. Intussusception in children: evidence-based diagnosis and treatment. Pediatr Radiol. 2009;39(Suppl 2):S140-3.

18. Andersen JF, Eklof O, Thomasson B. Large bowel volvulus in children. Review of a case material and the literature. Pediatr Radiol. 1981;11(3):129-38.

19. Atamanalp SS, Yildirgan MI, Basoglu M, Kantarci M, Yilmaz I. Sigmoid colon volvulus in children: review of 19 cases. Pediatr Surg Int. 2004;20(7):492-5.

20. Moore CJ, Corl FM, Fishman EK. CT of cecal volvulus: unraveling the image. AJR Am J Roentgenol. 2001;177(1):95-8.

21. McCarthy HJ, Tizard EJ. Clinical practice: diagnosis and management of Henoch-Schonlein purpura. Eur J Pediatr. 2010;169(6):643-50.

22. Epelman M, Daneman A, Navarro OM, Morag I, Moore AM, Kim JH, et al. Necrotizing enterocolitis: review of state-of-theart imaging findings with pathologic correlation. Radiographics. 2007;27(2):285-305.

23. Esposito F, Mamone R, Di Serafino M, Mercogliano C, Vitale $\mathrm{V}$, Vallone $\mathrm{G}$, et al. Diagnostic imaging features of necrotizing enterocolitis: a narrative review. Quant Imaging Med Surg. 2017;7(3):336-44.

24. Krishnamoorthi R, Ramarajan N, Wang NE, Newman B, Rubesova E, Mueller CM, et al. Effectiveness of a staged US and CT protocol for the diagnosis of pediatric appendicitis: reducing radiation exposure in the age of ALARA. Radiology. 2011;259(1):231-9.

25. Wan MJ, Krahn M, Ungar WJ, Caku E, Sung L, Medina LS, et al. Acute appendicitis in young children: cost-effectiveness of US versus CT in diagnosis-a Markov decision analytic model. Radiology. 2009;250(2):378-86.

26. Anupindi SA, Podberesky DJ, Towbin AJ, Courtier J, Gee MS, Darge K, et al. Pediatric inflammatory bowel disease: imaging issues with targeted solutions. Abdom Imaging. 2015;40(5):975-92.

27. Kotecha M, Bellah R, Pena AH, Jaimes C, Mattei P. Multimodality imaging manifestations of the Meckel diverticulum in children. Pediatr Radiol. 2012;42(1):95-103.

28. Sivit CJ. Imaging children with abdominal trauma. AJR Am J Roentgenol. 2009;192(5):1179-89.

29. Trout AT, Strouse PJ, Mohr BA, Khalatbari S, Myles JD. Abdominal and pelvic CT in cases of suspected abuse: can clinical and laboratory findings guide its use? Pediatr Radiol. 2011;41(1):92-8.

30. Sivit CJ, Taylor GA, Bulas DI, Kushner DC, Potter BM, Eichelberger MR. Posttraumatic shock in children: CT findings associated with hemodynamic instability. Radiology. 1992;182(3):723-6.

Open Access This chapter is licensed under the terms of the Creative Commons Attribution 4.0 International License (http://creativecommons. org/licenses/by/4.0/), which permits use, sharing, adaptation, distribution and reproduction in any medium or format, as long as you give appropriate credit to the original author(s) and the source, provide a link to the Creative Commons license and indicate if changes were made.

The images or other third party material in this book are included in the book's Creative Commons license, unless indicated otherwise in a credit line to the material. If material is not included in the book's Creative Commons license and your intended use is not permitted by statutory regulation or exceeds the permitted use, you will need to obtain permission directly from the copyright holder.

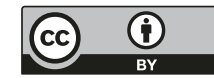

